\title{
Askin Tumor in Egyptian Patients; 5 Years Experience at the National Cancer Institute, Cairo University
}

\author{
Ahmed El Sayed Fathalla, Bahaa El Din Ahmed \\ National Cancer Institute, Cairo University, Giza, Egypt \\ Email: drasf1975@gmail.com, bahaaahmed897@yahoo.com
}

Received 28 January 2016; accepted 15 March 2016; published 18 March 2016

Copyright (C) 2016 by authors and Scientific Research Publishing Inc.

This work is licensed under the Creative Commons Attribution International License (CC BY). http://creativecommons.org/licenses/by/4.0/

(c) (i) Open Access

\section{Abstract}

Background: Askin tumor is a primitive neuroectodermal tumor of the Ewing sarcoma family arising from soft tissues of the chest wall. It is the commonest chest wall malignancy in children and adolescents. Its diagnosis is complex and management requires a multidisciplinary work including chemotherapy for systemic disease and radiation therapy to assist local control which is achieved through surgery with or without reconstruction. Objective: To analyze report and understand the clinicopathological features, results and outcome of this tumor with assessment of early and late postoperative complications following resection and chest wall reconstruction. Materials and Methods: This is a retrospective analysis of 30 cases with chest wall ES/PNET presented to the National Cancer Institute; Cairo University between January 2011 and December 2015. All patients' records were revised for age, sex, clinical presentation, imaging, pathology, operative notes, different treatment modalities given, early and late postoperative complications following surgical resection or reconstruction, and outcome including overall survival (OS) and disease free survival (DFS). Results: Our cohort included 30 patients with chest wall ES. The median follow up period $(n=30)$ was 33.7 months (ranging from 3.7 to 69.3 months). The median $0 S$ was 54.2 months with cumulative OS at 60 months which was $45.6 \%$. The median DFS was 27.9 months with cumulative DFS at 60 months which was 40.6\%. All cases were below 18 years with a male predominance $(n=19)$. The commonest affected sites were ribs $(n=22,73.3 \%)$, the scapula $(n=5,16.6 \%)$, the clavicle $(n=2,6.66 \%)$ and the sternum $(n=1,3.33 \%) .23$ patients $(76.6 \%)$ were presented with localized disease, and 7 patients $(23.4 \%)$ were metastatic from the start. All patients received neoadjuvant chemotherapy (4 cycles of VAC/IE) followed by local control: either surgery ( 26 cases, $86.6 \%$ ) or radical radiotherapy ( 3 cases, 10\%). A single case of a rib ES with initial bilateral lung deposited where no local control was done. After chest wall resection, closure of the defect was done by 1 ry closure with no reconstruction or double layer prolene mesh and bone cement that was covered by pedicled flap (latissimus dorsi, serratus anterior or pectoralis major muscle flap). Postoperative radiation therapy was given to 9 patients: 4 (13.3\%) had postopera- 
tive poor chemotherapy effect ( $<90 \%$ tumor necrosis), $3(10 \%)$ had +ve microscopic safety margin and $2(6.6 \%)$ had pleural based nodules with malignant pleural effusion at initial presentation. Conclusion: Askin's tumor shows a dramatic response to polychemotherapy. Treatment of such tumor should include multidisciplinary working groups for optimum results and better survival.

\section{Keywords}

\section{Chest Wall, Askin Tumor, Management \& Outcome}

\section{Introduction}

ES/PNET is the most common primary flat bone tumor. It is the 2nd most common malignant bone tumor in children and adolescents after osteosarcoma and the 3rd commonest malignant chest wall tumor (10\%). $15 \%$ of these ES arise in the chest wall and are named Askin's tumor [1] [2].

It was first described by James Ewing in 1921 after observing radiosensitivity in a subgroup of bone tumors. In 1979 Askin et al. described a unique clinicopathologic entity characterized as a malignant small cell tumor of the thoracopulmonary region in 20 children and adolescents [3].

These tumors affect males 1.5 times the females and appear to originate in the soft tissues of the chest wall or the peripheral lung. This ES family of tumors includes the classical bony ES, extraskeletal ES, Askin's tumor (ES of the thoracopulmonary region), and peripheral primitive neuroectodermal tumor (PNET) [3] [4].

ES family arises from cells of the primitive neural crest and all contain the same reciprocal translocation $\mathrm{t}$ (11, 22). They show a variable degree of neuroectodermal differentiation. The term Ewing sarcoma is used for tumors lacking the evidence of neuroectodermal differentiation assessed by light microscopy, immunohistochemistry, and electron-microscopy, whereas, the term PNET is used for those that demonstrate neuroectodermal features [3]-[5].

Askin tumors are often very large at the time of diagnosis. Pain is commonest manifestation as a result of the mass effect and is usually of several months duration. Additional symptomatology includes progressive shortness of breath, dyspnea, fever, night sweats, and weight loss [5] [6].

They are treated based on their clinical presentation (local disease or distant deposits) rather than the histologic subtype. Multimodality therapy has improved the survival results, and a long-term survival is frequently achieved after complete chest wall resection which may be facilitated by neoadjuvant chemotherapy. Whether adjuvant radiotherapy is beneficial remains unclear as long-term survival could be obtained without radiation therapy and consideration of radiation therapy should be made on a case-by-case basis. Initial treatment usually involves multidrug chemotherapy, as most tumors respond to this modality followed by surgical resection [7].

When the tumor arises from a rib, it is not fully clear whether resection of the rib with a wide tumor-free margin alone is adequate or resection of at least three complete ribs. Postoperative radiotherapy is used mainly in the situation when there is inadequate surgical resection margin; however, a major complication following radiation is the development of another tumor or cardiac and pulmonary injury. Unresectable tumors are treated mainly with chemotherapy followed by radiotherapy [7] [8].

Recurrence occurs in approximately $20 \%$ of patients and about $25 \%$ of patients are metastatic at presentation. The most important predictive factor for local recurrence is the response to chemotherapy. The 5-year survival for localized disease approaches $100 \%$ and is $30 \%$ for metastatic disease [8].

This study aims to analyze, to report and to understand the clinicopathological features, results and outcome of management with assessment of early and late postoperative complications following resection and chest wall reconstruction.

\section{Materials and Methods}

This is a retrospective analysis including all cases with chest wall ES/PNET presented to the National Cancer Institute, Cairo University between January 2011 and December 2015. Chest wall ES tumors include tumors that arise from the ribs, sternum, clavicle and the scapula. A total number of 30 cases were identified from the surgical pathology files and clinical information was obtained through reviewing of the medical reports. Each pa- 
tient record was revised for age, sex, clinical presentation, imaging, pathology, operative notes, different treatment options given, early and late postoperative complications after resection or reconstruction, and outcome analysis including OS and DFS. Treatment failure patterns were classified into local recurrence and distant metastasis. Ethical clearance for the conduction of this study was obtained from our institute ethical committee.

\section{Statistical analysis}

Overall survival (OS) was calculated as the time between date of diagnosis and date of death or last follow up for those alive till the end of the study. Disease free survival (DFS) was calculated as the time between date of surgery (cure) and date of relapse or death or last follow up for those alive free till the end of the study.

Data was analyzed using IBM SPSS advanced statistics version 22 (SPSS Inc., Chicago, IL). Follow up time was expressed as median and range. Qualitative data were expressed as frequency and percentage. Survival analysis was done using Kaplan-Meier method and presented as median survival with its 95\% confidence interval (CI) and the cumulative survival at 36 months and 60 months. Event of OS survival was death \& for DFS was relapse/death.

\section{Results}

Male predominance was found $(\mathrm{n}=19 \%)$ compared to females $(\mathrm{n}=11)$. The affected sites were ribs $(\mathrm{n}=22$, $73.3 \%)$, the scapula $(n=5,16.6 \%)$, the clavicle $(n=2,6.66 \%)$ and the sternum $(n=1,3.33 \%)$. All our cases were below 18 years at presentation.

23 patients (76.6\%) presented with localized disease with no evidence of distant disease and 7 patients (23.4\%) were metastatic from the start (6 cases with a 1 ry rib lesion and 1 case with 1 ry sternal lesion). The main sites of metastases were bone marrow infiltration (4 cases), malignant pleural based nodules with malignant effusion ( 2 cases) and one case initially presented with bilateral pulmonary metastases.

All our patients received neoadjuvant chemotherapy in the form 4 cycles of vincristine, adriamycin, cyclophosphamide (VAC)/iphosphamide, etoposide (IE) with a 3 weeks interval between each cycle followed by local control either by surgical resection or radical dose of radiotherapy, except a single case of a rib ES which initially presented with bilateral pulmonary metastases where no local control was done. 26 cases (86.6\%) underwent local control by surgical resection and 3 cases (10\%) of scapular ES achieved local control by radical dose of radiation therapy (Figure 1, Figure 2).

26 cases underwent surgical resection. The extent of resection in this study was shown in (Table 1) and (Figure 3).

After performing chest wall resection, there were different methods used for closure of the defect as shown in Table 2.

Postoperative radiation therapy was given to 9 patients, 4 of them (13.3\%) had evident postoperative poor chemotherapy effect (<90\% tumor necrosis), $3(10 \%)$ had +ve microscopic surgical margin and 2 cases (6.6\%) had pleural based nodules with malignant pleural effusion at initial presentation.
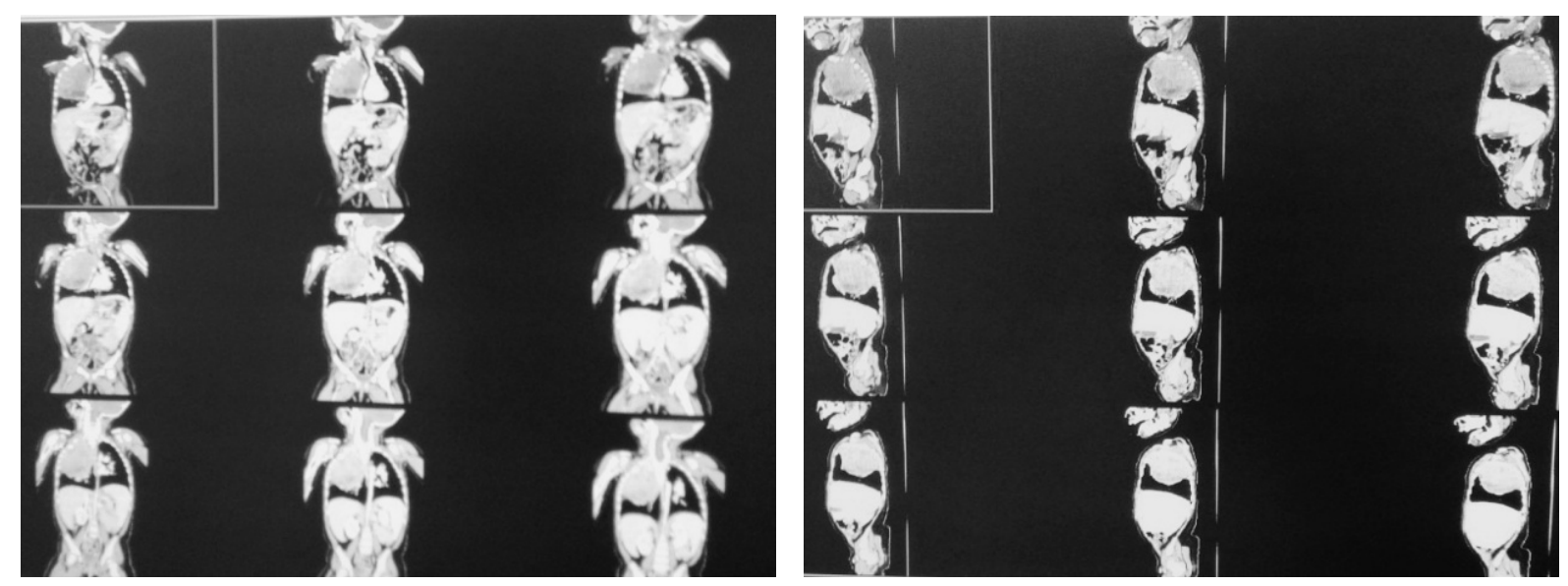

Figure 1. CT scan (coronal and axial views) of one of our patients with right upper rib Ewing at the initial presentations. 


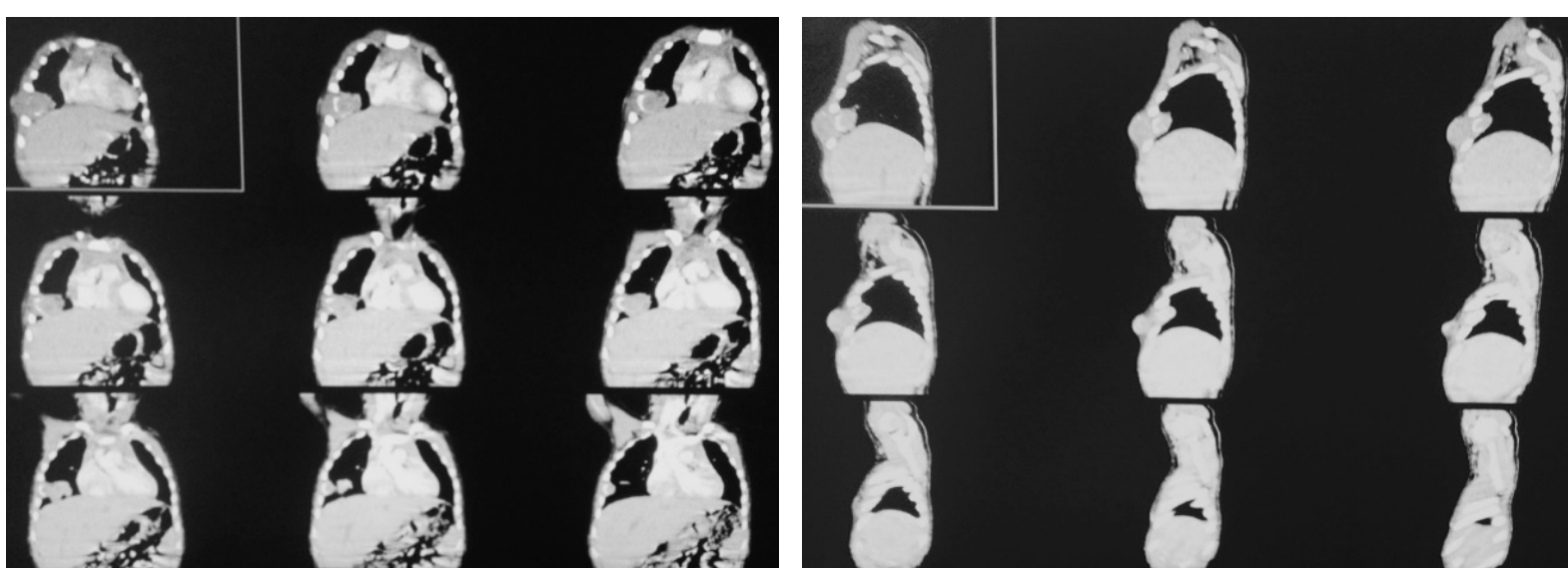

Figure 2. CT scan (coronal \& axial views) of another patient with right lower rib Ewing at the initial presentation.
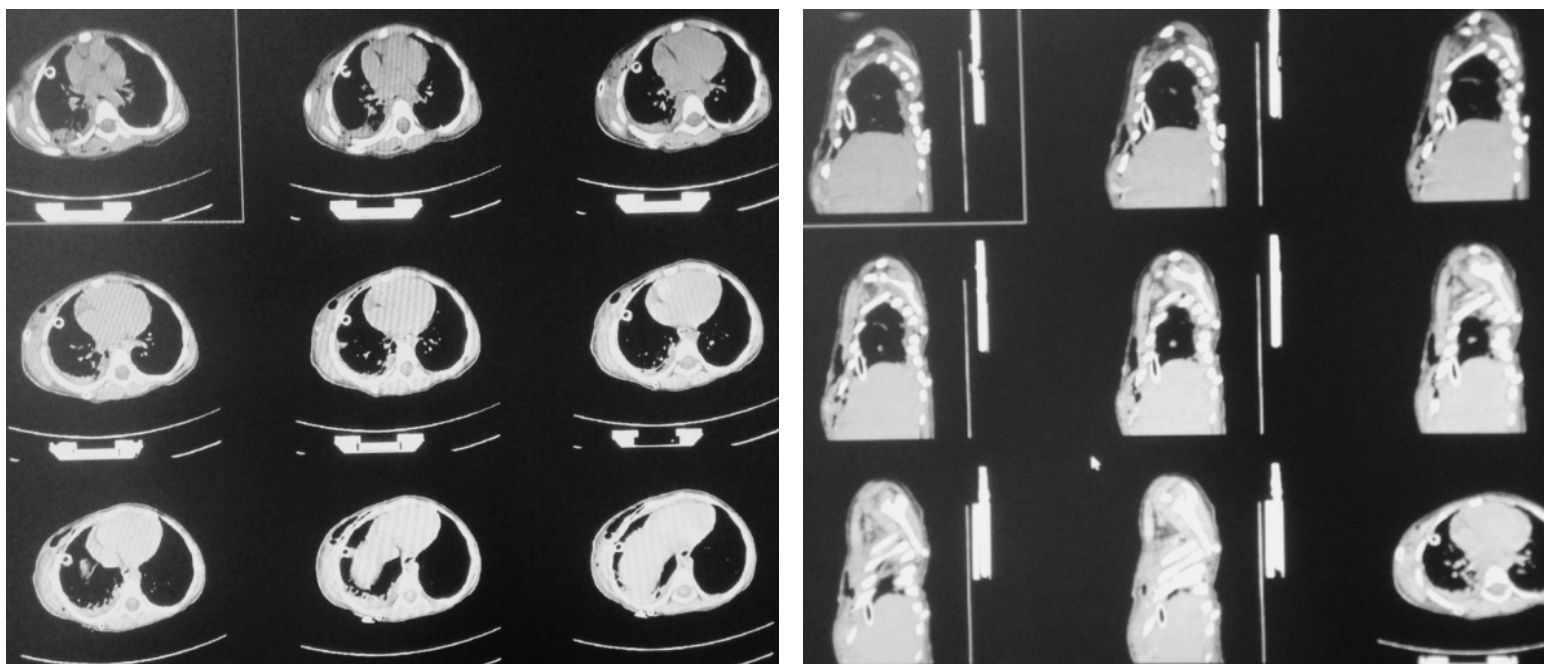

Figure 3. CT scan (axial \& saggittal views) during the early (day 2) postoperative period after rib resection.

Table 1. Extent of resection.

\begin{tabular}{cc}
\hline Site \& No. & Parts resected \\
\hline & Resection of 1 Rib (4 cases) \\
\hline
\end{tabular}

Resection of 2 Ribs (11 cases)

Resection of 3 Ribs (6 cases)

Ribs (22 cases*)

Resection of part of the diaphgram (3 cases)

Segmental resection of the lung (4 cases)

Resection of the Xiphoid process and part of the diaphgram (1 case)

Clavicle (2 cases)

Total right clavilectomy (2 cases)

Scapula (5 cases*)

Subtotal scapulectomy (2 cases)

Sternum (1 cases) Resection of the upper part of the sternum + upper costal cartilages

"There was one case of rib Ewing's sarcoma that didn't have local control as it was presented by bilateral pulmonary deposits at the time of diagnosis. There were also 3 cases of scapular ES that underwent local control by radical dose of radiotherapy in a dose of 5600:5800 CGY. 
The median follow up period for the PENT patients $(n=30)$ was 33.7 months (range: 3.7 - 69.3 months). The median overall survival (OS) was 54.2 months with cumulative OS at 36 and 60 months of $60.7 \%$ and $45.6 \%$ respectively. The median disease free survival (DFS) was 27.9 months with cumulative DFS at 36 and 60 months of $48.7 \%$ and $40.6 \%$ (Figure 4 ).

\section{Discussion}

Many theories have evolved regarding the origin of ES family of tumors, the two theories with the most support suggest that these tumors arise from a primitive cell derived either from an embryologic tissue (the neural crest), or from resident cells in the body (mesenchymal stem cells). 90\% of Askin tumor cases occur between the ages of 5 and 25 years. Males are affected 1.5 times with poorer survival than females with the white race 10 times more affected than the blacks. All our cases were below 16 years with males affected 1.7 times [8] [9].

Patients usually present with chest wall mass, cough, chest pain that is usually vague and diffuse due to periosteal or neural invasion, respiratory distress from massive malignant effusion or less commonly from extensive intra thoracic involvement, fever (remittent, low grade), anemia, leucocytosis, eosinophilia and increased ESR, night sweat and weight loss. The most common clinical features in this study were palpable chest wall mass and frequent chest pain on the ipsilateral tumor site [10].

Radiographic appearance of ES in the thorax is quite variable and the lesion may be primarily lytic, sclerotic or mixture of both. A universe feature of the disease is the presence of a large soft tissue mass relatively larger than the extent of bone changes. Sometimes the only apparent change in a radiograph is the presence of a mass, cortical erosion, or periosteal reaction being hardly visible. In this work, the radiologic modality of choice was

\begin{tabular}{lcc}
\hline \multicolumn{2}{l}{ Table 2. Methods of postoperative reconstruction. } \\
\hline Site and No. & Method of reconstruction & 26 cases \\
\hline & $\begin{array}{c}\text { Double layer prolene mesh + bone cement + pedicled LDMF } \\
\text { Ribs (21) }\end{array}$ & 4 \\
& $\begin{array}{r}\text { Double layer prolene mesh + bone cement + coverage by local muscle } \\
\text { Double layer prolene mesh covered by local muscle }\end{array}$ & 5 \\
Scapula (5) & Refixation of the scapula after extracorporeal RTH & 3 \\
Clavicle (2) & Only resection (no reconstruction) & 2 \\
Sternum (1) & $\begin{array}{l}\text { Double layer prolene mesh + bone cement } \\
\text { + bilateral pectoralis major muscle flap }\end{array}$ \\
\hline
\end{tabular}
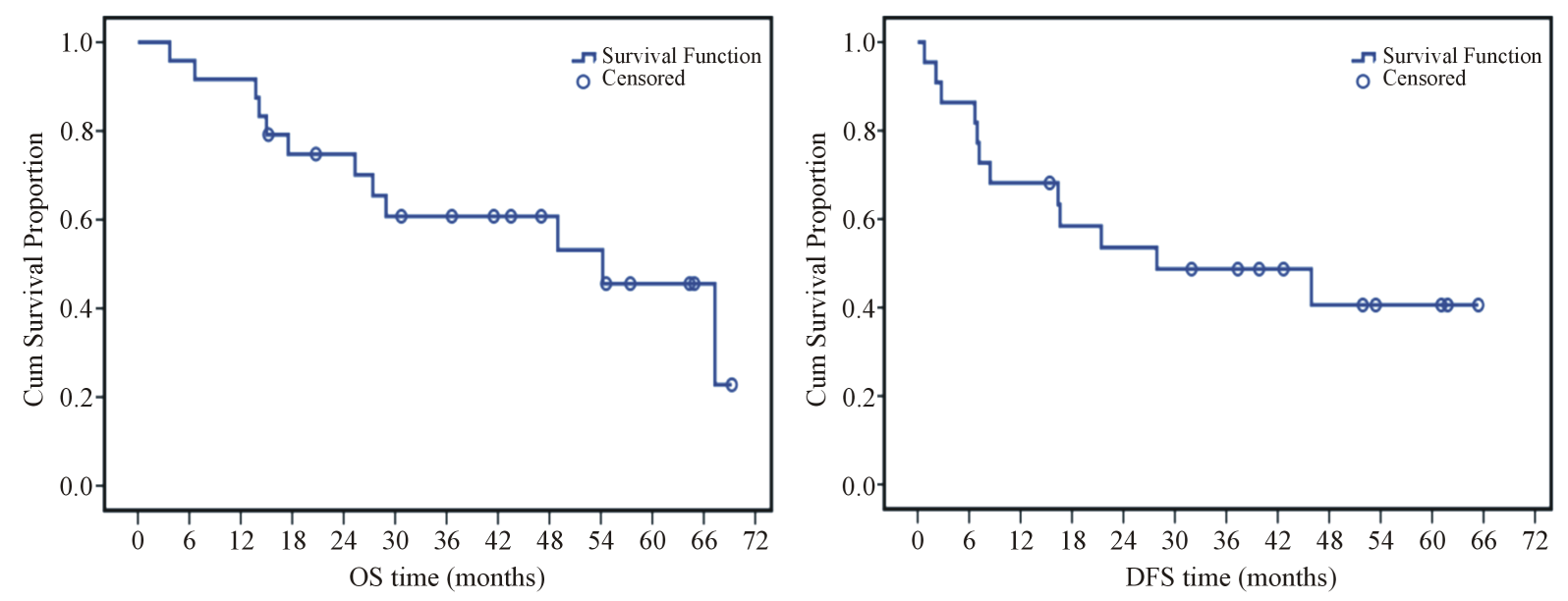

Figure 4. OS and DFS curves (P value $<0.05$ was considered significant). 
CT scanning of the chest wall (Figures 1-3); it was helpful for identifying cortical breakthrough, determining the extent of extra osseous tumor extension, and evaluating primary extra osseous tumors. CT scan was also helpful in detecting pulmonary metastases, and was superior to PET scan (spiral CT scanning) for detecting pulmonary metastases. Tissue biopsy is the corner stone of diagnosis, both in our study and the literature. A controversy still exists regarding the radiologic modality of choice whether it is CT scanning or MRI. MRI may be helpful for paraspinal lesions; delineating relations to critical structures, helpful to monitor the response to neoadjuvant CTH and for follow up after tumor resection [11].

The standard therapy for chest wall ES/PNET is neoadjuvant chemotherapy followed by local control with surgical resection with or without radiotherapy. Two standard regimens of chemotherapy are used; the 3 drug regimen (Cyclophosphamide, Actinomycin-D and Vincristine) and the 5 drug regimen (Vincristine, Doxorubicin, Cyclophosphamide, Ifosfamide and Etoposide). Actinomycin-D is no longer used in the United States but continues to be used in the Euro-Ewing studies and is replaced instead by Doxorubicin. Patients receiving 5 drug therapy had improved survival compared to those receiving 3 drug therapy (overall survival $72 \%$ vs. $61 \%, \mathrm{P}=$ 0.01). In this work all our patients received the 5 drug regimen with median overall survival (OS) of 54.2 months and cumulative OS at 36 and 60 months of $60.7 \%$ and $45.6 \%$ respectively. The 5 drug therapy in our study included 4 cycles with 3 weeks interval between each cycle before local control where All our patients received chemotherapy first with delayed surgery [12] [13].

Local control in our study was achieved by surgical resection with postoperative radiation therapy given in cases with +ve microscopic margin, initial malignant pleural effusion, pleural based nodules and poor CTH effect (less than $90 \%$ tumor necrosis). We obtained local control in this study following the neoadjuvant protocol even with resection of nearby structures [13]-[15].

Chest wall reconstruction is needed only if there is resection of three ribs or more. The goals of the reconstruction are adequate stability, water and airtight closure of the chest cavity, and acceptable cosmetic appearance. In our study, chest wall reconstruction was done by the use of double layer prolene mesh with or without bone cement that is covered either by rotational pedicled muscle flap (latissimus dorsi or serratus anterior) or covered by the local muscle flap (pectoralis major) [14] [15].

Postoperative complications include unintentional lung collapse (pneumothorax), nerve damage, fracture along the rib in other locations, perforation of the diaphragm, wound infection that occurs in about $10 \%-15 \%$ of cases, and pneumonia. In our study, the main complication was atelectases which occurred in all our patients and resolved always after 48 hours of surgery. There was no early or late infection related to the use prolene mesh used. 2 cases with rib resection after reconstruction of the chest wall by prolene mesh and local muscle flap suffered laxation of the mesh and some flail chest which required redo operation with tightening of the original mesh only [14] [15].

Rehabilitation and physical therapy after rib resection is mandatory to decrease pain, prevent respiratory complications and restore function. All our patients were encouraged to start physical starting from the first day postoperatively for those who can use the spirometer (above the age of 5 in our study). For those below this age physical therapy was done mainly by allowing child movement freely and light regular percussion on the back [15].

Adjuvant radiation therapy is indicated in poor CTH effect (less than $90 \%$ tumor necrosis), positive microscopic surgical margin, initial malignant pleural effusion and/or malignant based pleural nodules. Radiation therapy is better avoided in children because of its potential complications especially, chronic swelling, skin complications, pulmonary fibrosis, increased risk of doxorubicin induced cardiomyopathy and 2 ry malignancies later in life in less than $5 \%$ of cases. Those patients who received $60 \mathrm{~Gy}$ or more had an incidence of $2^{\text {nd }}$ malignancy of 20\%. In our study 3 patients received radiotherapy for microscopic positive surgical margin, 4 patients for poor CTH effect and 2 patients for pleural based nodules at initial presentation and initial malignant pleural effusion [16].

\section{Conclusion}

Chest wall ES/PNET mainly affects young age ( $<18$ years). The commonest site of affection is the ribs. The majority of cases present with large sized chest wall mass; thus neoadjuvant chemotherapy offers the opportunity to downsize the tumor and allow resection with negative margins which avoid postoperative radiotherapy in good responders to chemotherapy. Resection resulting in large defects, reconstruction with prolene mesh cov- 
ered by muscle flap will provide effective coverage without significant complications.

\section{References}

[1] Davidson, M.J. (1991) Ewing’s Sarcoma of the Temporal Bone. A Case Report. Oral Surgery, Oral Medicine, Oral Pathology, 72, 534-536. http://dx.doi.org/10.1016/0030-4220(91)90489-Y

[2] Watanabe, H., Tsubokawa, T., Katayama, Y., et al. (1992) Primary Ewing's Sarcoma of the Temporal Bone. Surgical Neurology, 37, 54-58. http://dx.doi.org/10.1016/0090-3019(92)90067-w

[3] Marina, N.M., Etcubanas, E., Parman, D.M., Bowman, L.C. and Green, A. (1989) Peripheral Primitive Neuroectodermal Tumor (Peripheral Neuro-Epithelioma) in Children: A Review of the St Jude Experience and Controversies in Diagnosis and Management. Cancer, 64, 1952-1960.

http://dx.doi.org/10.1002/1097-0142(19891101)64:9<1952::AID-CNCR2820640931>3.0.CO;2-W

[4] Applebaum, M.A., Worch, J., Matthay, K.K., et al. (2011) Clinical Features and Outcomes in Patients with Extra Skeletal Ewing Sarcoma. Cancer, 117, 3027-3032. http://dx.doi.org/10.1002/cncr.25840

[5] Fletcher, C.D.M., Unni, K. and Mertens, F. (2002) Pathology and Genetics of Tumors of Soft Tissue and Bone. In: World Health Organization Classification of Tumors, IARC Press, Lyon, 26-27.

[6] Indelicato, D.J., Keole, S.R., Shahlaee, A.H., et al. (2008) Long-Term Clinical and Functional Outcomes after Treatment for Localized Ewing Tumor of the Chest Wall. International Journal of Radiation Oncology*Biology*Physics, 70 , 501-509. http://dx.doi.org/10.1016/j.ijrobp.2007.06.032

[7] Lin, P.P., Jaffe, N., Herzog, C.E., et al. (2007) Chemotherapy Response Is an Important Predictor of Local Recurrence in Ewing Sarcoma. Cancer, 109, 603-611. http://dx.doi.org/10.1002/cncr.22412

[8] Womer, R.B., West, D.C., Kralio, M.D., et al. (2012) Randomized Controlled Trial of Interval-Compressed Chemotherapy for the Treatment of Localized Ewing Sarcoma: A Report from the Children Oncology Group. Journal of Clinical Oncology, 30, 4148-4154. http://dx.doi.org/10.1200/JCO.2011.41.5703

[9] Randall, L., Calvert, G., Sparker, H., et al. (2011) Ewing’s Sarcoma Family of Tumors (ESFT). The Liddy Shriver Sarcoma Initiative, 8.

[10] Shamberger, R.C., Laquaglia, M.P., Gebhardt, M.C., et al. (2003) Ewing’s Sarcoma/Primitive Neuroectodermal Tumor of the Chest Wall. Annals of Surgery, 238, 563-568.

[11] Kleis, M., Daldrup-Link, H., Matthay, K., Goldsby, R., Lu, Y., Schuster, T., et al. (2009) Diagnostic Value of PET/CT for the Staging and Restaging of Pediatric Tumors. European Journal of Nuclear Medicine and Molecular Imaging, 36, 23-36. http://dx.doi.org/10.1007/s00259-008-0911-1

[12] Grier, H.E., Krailo, M.D., Tarbell, N.J., et al. (2003) Addition of Ifosfamide and Etoposide to Standard Chemotherapy for Ewing's Sacoma and Primitive Neuroectodermal Tumor of Bone. New England Journal of Medicine, 348, 694-701. http://dx.doi.org/10.1056/NEJMoa020890

[13] Shamberger, R.C., et al. (2003) Ewing Sarcoma/Primitive Neuroectodermal Tumor of the Chest Wall: Impact of Initial versus Delayed Resection on Tumor Margins, Survival, and Use of Radiation Therapy. Annals of Surgery, 238, 563567, Discussion 567-568. http://dx.doi.org/10.1097/01.sla.0000089857.45191.52

[14] Grosfeld, J.L., et al. (1998) Chest Wall Resection and Reconstruction for Malignant Conditions in Childhood. Journal of Pediatric Surgery, 23, 667-673. http://dx.doi.org/10.1016/S0022-3468(88)80642-0

[15] Onder, B. and Hasan, H. (2007) Extremity Saving Surgery and Reconstruction for Tumors of the Scapula. Acta Orthopaedica et Traumatologica Turcica, 41, 195-201.

[16] Wagner, T.D., Kobayashi, W., Dean, S., et al. (2009) Combination Short Course Preoperative Irradiation, Surgical Resection, and Reduced-Field High-Dose Postoperative Irradiation in the Treatment of Tumors Involving the Bone. International Journal of Radiation Oncology*Biology*Physics, 73, 259-266. http://dx.doi.org/10.1016/j.ijrobp.2008.03.074 